\title{
MODELO DE PREDICCIÓN DE RESPUESTAS CARDIOVASCULARES DURANTE LA INMERSIÓN EN UN ENTORNO ACUÁTICO
}

\author{
D. Martínez-Pascual, M. Arnau-Papí, J.M. Catalán, L.D. Lledó, S. Ezquerro, F.J. Verdú-García, Nicolás García-Aracil \\ Universidad Miguel Hernández de Elche, Instituto de Bioingenieria, david.martinezp@umh.es
}

\section{Resumen}

El sistema cardiovascular se ve afectado cuando el cuerpo humano se sumerge en un entorno acuático. Para estudiar y predecir las respuestas del sistema cardiovascular durante la inmersión se ha desarrollado un modelo de alto nivel. El modelo presentado se compone de un conjunto de ecuaciones en diferencias que describe el comportamiento del sistema cardiovascular de acuerdo con el nivel de inmersión. El modelo se evalúa en simulación y se compara con los efectos descritos en la literatura científica en sesiones experimentales.

Palabras clave: Modelado sistema cardiovascular, robótica de rehabilitación, rehabilitación acuática

\section{INTRODUCCIÓN}

Diferentes trastornos motores provocados por ictus, lesiones medulares o esclerosis múltiple pueden traer discapacidades crónicas que limiten la autonomía y calidad de vida. En pacientes de ictus la espasticidad es un síntoma muy común, afectando hasta el $30 \%$ de los pacientes [15]. Además, se ha demostrado que la prevalencia de la espasticidad no difiere entre miembros superiores e inferiores. En el caso de pacientes con lesiones medulares la espasticidad llega a afectar hasta el $70 \%$ de los sujetos, causando un grado de discapacidad considerable [12]. Asimismo, el desarrollo de contracturas y rigidez en las articulaciones es una de las secuelas más importantes en pacientes de lesiones medulares. En concreto, se han reportado contracturas en al menos una articulación para el $66 \%$ de los pacientes [10].

La robótica de rehabilitación es una disciplina que puede realizarse como un complemento de las terapias convencionales, y cuyo propósito es asistir el movimiento de los miembros afectados empleando sistemas robóticos para tratar de disminuir las discapacidades motoras [8]. No obstante, diversos autores señalan que, tanto para los miembros superiores como inferiores, algunos pacientes fueron excluidos de las pruebas de control debido a una espasticidad severa, dolor o rango de movimiento limitado, entre otros factores [17]. Para tratar de superar estos problemas, y poder ofrecer esta terapia a los pacientes excluidos en las pruebas, una posibilidad sería realizar la terapia de rehabilitación en un tanque de agua con unas condiciones controladas. Realizar la terapia de forma sumergida en este tipo de entorno puede aportar ciertas ventajas como una descarga de las articulaciones del paciente o una menor resistencia al movimiento, y ha demostrado ser una terapia efectiva para tratar la artritis [14] o las lesiones medulares [6].

El proyecto SPLASH nace como un intento para superar estos problemas y ofrecer terapia de rehabilitación con dispositivos robóticos a los pacientes excluidos. El principal objetivo del proyecto SPLASH es investigar un paradigma de rehabilitación radicalmente nuevo. El paradigma propuesto va más allá del desarrollo de dispositivos robóticos sumergibles, ya que se propone estudiar los efectos del cuerpo humano en un entorno acuático cambiante. Por ello, para tener una mejor comprensión de las reacciones del cuerpo humano, se propone desarrollar modelos de alto nivel para estimar el estado del usuario en el entorno de apoyo. Sin embargo, existe una falta de modelos matemáticos que describan las respuestas fisiológicas del cuerpo humano en un entorno acuático cambiante.

Cabe destacar que diversos autores han demostrado que el sistema cardiovascular se ve afectado cuando el cuerpo se encuentra sumergido. Además, las respuestas fisiológicas varían de acuerdo con el nivel de inmersión, tal y como recogen I. M. Wilcock et al. [18]. En concreto, se ha demostrado que estos cambios se deben principalmente a una redistribución de la sangre desde los miembros al tórax, especialmente desde los miembros inferiores [13].

En este trabajo se presenta un modelo de alto nivel que describe las respuestas del sistema cardiovascular en un entorno acuático a temperatura termoneutral, con un nivel de inmersión cambiante. 


\section{MÉTODOS}

\subsection{ANTECEDENTES}

La literatura científica recoge diferentes modelos para estudiar las respuestas del sistema cardiovascular bajo ciertas condiciones. Prueba de ello es el modelo desarrollado por S. Timischl [16], un modelo en tiempo continuo que describe el comportamiento del sistema cardiovascular durante el ejercicio físico. No obstante, otros autores prefieren no despreciar la naturaleza pulsátil del corazón. Ejemplos de ello son el modelo del sistema cardiovascular desarrollado por S. Gisler para escenarios de rehabilitación [2], o el modelo desarrollado por R. DeBoer et al., el cual describe el comportamiento del sistema cardiovascular durante el reposo [1]. Ambos autores proponen modelos latido a latido compuestos por un conjunto de ecuaciones en diferencias.

A pesar de que se han desarrollado modelos que explican el comportamiento del sistema cardiovascular en diferentes situaciones, existe una falta de modelos capaces de describir las respuestas del sistema cardiovascular durante la inmersión en un entorno acuático. Por tanto, el modelo matemático propuesto está basado en los efectos que produce la inmersión, recogidos en la bibliografía. En concreto, el modelo se basa en los datos experimentales recabados por K. S. Park et al. [11] y la revisión sistemática realizada por I. M. Wilcock et al.

La mayoría de modelos del sistema cardiovascular están compuestos por reservas o compartimentos que representan las partes que lo forman. El modelo propuesto por S. Gisler es una muestra de ello, y en cuyo modelo nos basamos. Asimismo, los modelos que explican el funcionamiento completo del sistema cardiovascular suelen incluir elementos del sistema nervioso. Suele ser común incluir el reflejo barorreceptor debido a que regula la presión sanguínea y el ritmo cardíaco a corto plazo. Por tanto, el modelo matemático propuesto incorpora el reflejo barorreceptor para así cerrar el bucle de control del sistema, y está basado en el modelo propuesto por R. DeBoer et al. En la Figura 1 se muestra un esquema del modelo propuesto.

Siguiendo la filosofía de los modelos latido a latido, se propone un modelo en tiempo discreto, donde $k$ representa un latido. Esto provoca que las variables introducidas se actualicen en cada latido. No obstante, cabe destacar que cada una de las características del sistema cardiovascular se actualiza en determinados instantes de tiempo durante el ciclo cardiaco, como se muestra en la Figura 2.

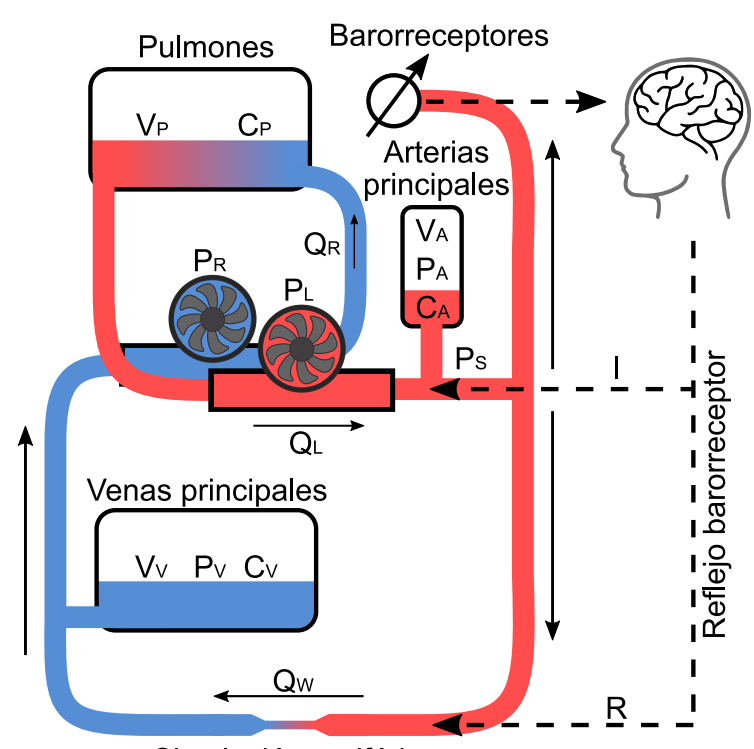

Circulación periférica

Figura 1: Representación simplificada del sistema cardiovascular del ser humano.

\subsection{MODELADO DEL SISTEMA CARDIOVASCULAR}

\subsubsection{Hemodinámica}

En el modelo propuesto se tiene en cuenta la circulación en las arterias y venas principales, pero también la circulación pulmonar y la microcirculación en partes periféricas del cuerpo. Esta microcirculación se modela como un estrechamiento de los vasos sanguíneos y se conoce como resistencia periférica. Además, cabe destacar que los vasos sanguíneos no se modelan como tubos rígidos, ya que se modelan como vasos con una cierta elasticidad.

El modelo propuesto por S. Gisler está formado por 3 reservas principales, y representan el sistema pulmonar, las arterias y las venas. Cada reserva tiene asociado un volumen $\left(V_{P}, V_{A}, V_{V}\right)$, una determinada elasticidad $\left(C_{P}, C_{A}, C_{V}\right)$ y una constante que representa el volumen sanguíneo no estresado $\left(V_{P 0}, V_{A 0}, V_{V 0}\right)$. Los volúmenes estresados causan que la sangre circule a unas determinadas presiones, que pueden definirse como

$$
\begin{aligned}
& P_{R}(k)=\frac{V_{V}(k-1)-V_{V 0}}{C_{V}(k)} \\
& P_{L}(k)=\frac{V_{P}(k-1)-V_{P 0}}{C_{P}}
\end{aligned}
$$




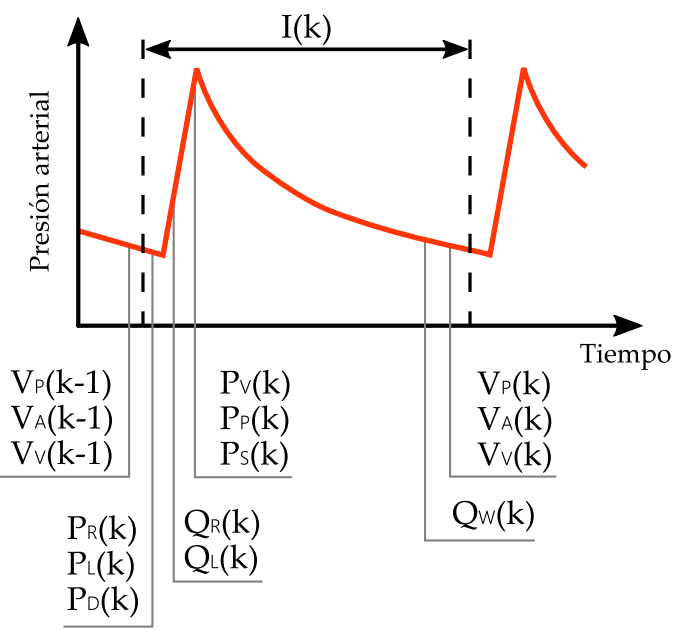

Figura 2: Instante de actualización de variables hemodinámicas durante el ciclo cardiaco

$$
P_{D}(k)=\frac{V_{A}(k-1)-V_{A 0}}{C_{A}}
$$

donde $P_{R}$ y $P_{L}$ representan las presiones en las aurículas derecha e izquierda, y $P_{D}$ la presión diastólica, que depende directamente del volumen sanguíneo en las arterias.

Nótese que $C_{P}$ y $C_{A}$ se modelan como constantes, pero $C_{V}$ se expresa como una variable. De acuerdo con K. S. Park et al., la compresión hidrostática reduce la elasticidad de las venas, especialmente en los miembros inferiores. Por tanto, $C_{V}$ se expresa como función del nivel de inmersión. Una posible aproximación es considerar el término dependiente de la inmersión como aditivo

$$
C_{V}(k)=C_{V 0}-\delta_{V} \cdot \frac{H_{b}(k)}{H_{H}}
$$

donde $C_{V 0}$ indica la elasticidad de las venas sin inmersión, $H_{b}$ el nivel de inmersión, $H_{H}$ la altura del sujeto y $\delta_{V}$ un factor de proporcionalidad.

Además, el flujo entre reservas puede expresarse de forma general como

$$
V_{j}(k)-V_{j}(k-1)=Q_{\text {in }}(k)-Q_{\text {out }}(k)
$$

siendo $Q_{\text {in }}$ y $Q_{\text {out }}$ las entradas y salidas de volúmenes de la reservas.

De acuerdo con S. Gisler, los volúmenes sistólicos izquierdo y derecho $\left(Q_{L}, Q_{R}\right)$, basados en la ley de Frank-Starling, pueden explicarse como una función de la precarga y la longitud del intervalo R-R (I) previo. Asimismo, de acuerdo con I. M. Wilcock et al., el volumen sistólico tiende a aumentar conforme aumenta el nivel de inmersión.
Este comportamiento se expresa en la ecuación (6) para los volúmenes sistólicos izquierdo y derecho $(R, L)$, donde $\gamma_{R, L}$ se trata de un factor constante, y $\delta_{R, L}$ un factor de proporcionalidad conforme al nivel de inmersión.

$$
\begin{aligned}
Q_{R, L}(k)=\gamma_{R, L} \cdot P_{R, L}(k) \cdot & I(k-1) \\
& +\delta_{R, L} \cdot \frac{H_{b}(k)}{H_{H}}
\end{aligned}
$$

Según S. Gisler, debido al efecto Windkessel producido en las arterias, la circulación periférica $\left(Q_{W}\right)$ depende de la diferencia de presión sistólica $\left(P_{S}\right)$ y la presión en las venas $\left(P_{V}\right)$, además de la longitud de $I$ y la resistencia periférica $(R)$.

$$
\begin{aligned}
& Q_{W}(k)=C_{A} \cdot\left(P_{S}(k)-\right.\left.P_{V}(k)\right) \\
& \cdot\left(1-e^{-\frac{I(k)}{R(k) \cdot C_{A}}}\right)
\end{aligned}
$$

En (7), $P_{V}$ hace referencia a la presión venosa justo después de la sístole, cuando $Q_{R}$ entra en la circulación pulmonar, lo que puede formularse como

$$
P_{V}(k)=\frac{V_{V}(k-1)-V_{V 0}-Q_{R}(k)}{C_{V}(k)}
$$

Las ecuaciones (9) y (10) definen la presión de pulso $\left(P_{P}\right)$, así como su relación con el volumen sistólico y la elasticidad arterial.

$$
\begin{gathered}
P_{P}(k)=P_{S}(k)-P_{D}(k) \\
P_{P}(k)=\frac{Q_{L}(k)}{C_{A}}
\end{gathered}
$$

\subsubsection{Regulación de la presión sanguínea}

La regulación de la presión sanguínea se lleva a cabo por regulaciones a corto plazo (segundos, minutos y horas), pero también por regulaciones a largo plazo (días o semanas). Para evaluar las respuestas del sistema cardiovascular durante la inmersión, solo se consideran las regulaciones a corto plazo.

En el modelo propuesto, el reflejo barorreceptor es responsable de regular la presión sanguínea, cerrando el bucle de control del sistema. Se sabe que el reflejo barorreceptor responde de forma proporcional en un rango limitado de presiones, cuya sensibilidad puede describirse como una función sigmoide [7]. Basándonos en el modelo 
desarrollado por R. DeBoer et al., esta función sigmoide puede expresarse como

$$
P_{B S}(k)=P_{B 0}+18 \cdot \arctan \left(\frac{P_{B}(k)-P_{B 0}}{18}\right)
$$

siendo $P_{B}$ una aproximación de la presión arterial media (MAP), calculada en (12). $P_{B S}$ es la MAP percibida por los barorreceptores y $P_{B 0}$ es el punto de equilibrio, que se considera la MAP del sujeto sin inmersión.

$$
P_{B}(k)=\frac{1}{3} P_{S}(k)+\frac{2}{3} P_{D}(k)
$$

A partir de una hipotética señal del reflejo barorreceptor, el sistema nervioso regula el ritmo cardiaco de acuerdo con la presión sanguínea. El modelo propuesto tiene en cuenta las acciones del sistema nervioso simpático y parasimpático en la regulación del ritmo cardiaco. Este comportamiento se expresa en (13), donde $I$ se determina a partir de $P_{B S}$ y $P_{P}$ durante el latido actual (debido a la influencia rápida del sistema parasimpático), junto con una suma ponderada de $P_{B S}$ de latidos previos (influencia simpática, más lenta), más una constante $K_{B}$.

$$
\begin{aligned}
I(k)=\alpha \cdot P_{B S}(k)+ & \sum_{n=2}^{6}\left(\beta_{I}(n) \cdot P_{B S}(k-n)\right) \\
& +K_{P} \cdot P_{P}(k)+K_{B}
\end{aligned}
$$

El reflejo barorreceptor también regula la presión sanguínea ajustando $R$. De acuerdo con R. DeBoer et al., las acciones de regulación de $R$ las lleva a cabo el sistema nervioso simpático. Esto también se expresa como una suma ponderada de $P_{B S}$ en ciclos previos, como se muestra en (14). Además, de acuerdo con I. M. Wilcock et al., diversos autores han demostrado que existe un decremento de $R$ con la inmersión. Este comportamiento se modela como un término negativo con una ganancia $\delta_{B R}$.

$$
\begin{array}{r}
R(k)=R_{P}-\sum_{n=2}^{6}\left(\beta_{R}(n) \cdot P_{B S}(k-n)\right) \\
-\delta_{B R} \cdot \frac{H_{b}(k)}{H_{H}}
\end{array}
$$

\subsection{DETERMINACIÓN DE PARÁMETROS}

En el modelo del sistema cardiovascular descrito existe una serie de constantes que será necesario determinar. Para determinar algunas de esas constantes nos basaremos en datos de la literatura científica, pero existen otros parámetros que será necesario determinar matemáticamente, empleando el modelo presentado.

Los volúmenes de sangre no estresados $V_{P 0}, V_{A 0} \mathrm{y}$ $V_{V 0}$ se determinan a partir de una aproximación de la distribución de los volúmenes de sangre en el cuerpo humano. De acuerdo con [4], del volumen sanguíneo total del cuerpo humano, un $9 \%$ se encuentra en el circuito pulmonar, un $64 \%$ en las venas, un $13 \%$ en las arterias, un $7 \%$ en el corazón y el $7 \%$ restante en capilares y vénulas. Además, se estima que aproximadamente el $57 \%$ del volumen sanguíneo corresponde a volumen no estresado [3]. Por tanto, una posible aproximación de volúmenes no estresados pasaría por aplicar la distribución sanguínea al cálculo del volumen no estresado. Asimismo, para calcular estos volúmenes se estimará el volumen total sanguíneo de un sujeto en base a su masa corporal, altura y sexo [9].

Para las ponderaciones de las acciones simpática y parasimpática en la regulación de $I$ y $R$, se emplea la ponderación triangular propuesta por R. DeBoer et al. Se empleará una ponderación triangular $\beta_{I}(n)=\beta_{R}(n)=[1,2,3,2,1]$ $\mathrm{ms} / \mathrm{mmHg}$ para los instantes $n=[k-2, k-3, k-$ $4, k-5, k-6]$.

En cuanto a la elasticidad de los vasos, $C_{P}, C_{A}$ y $C_{V 0}$ se ajustan para tener valores fisiológicos normales. Los valores empleados se asemejan en gran medida a los empleados por S. Gisler.

El resto de parámetros del modelo se calculan en el régimen permanente para dos condiciones: inmersión a una determinada altura y no inmersión. Para determinar estas constantes del modelo, establecemos valores de pulso y presiones sistólica y diastólica para ambas condiciones de inmersión. Estos datos están basados en la sesión experimental realizada por K. S. Park et al., y se recogen en la Tabla 1.

Para determinar todas las constantes del modelo, a cada condición se le impondrá dos restricciones adicionales. La primera restricción se expresa en (15), donde se fuerza a que la suma de volúmenes sea igual al volumen sanguíneo total (el 14\% del volumen total representa la sangre en capilares y corazón). En la segunda restricción se impone el valor de $R$ calculado en (16), siendo una aproximación de la resistencia total periférica [5]. Además, de acuerdo con [13], al sumergir el cuerpo humano se redistribuyen unos $500 \mathrm{~mL}$ de los miembros inferiores al circuito pulmonar, y esto se expresa en (17). 


$$
V_{T}=V_{P}+V_{A}+V_{V}+0.14 \cdot V_{T}
$$

$$
\begin{gathered}
R=\frac{P_{B}}{Q_{L} \cdot H R} \\
V_{P}=V_{\text {Pout }}+500[\mathrm{ml}]
\end{gathered}
$$

Imponiendo estas restricciones en régimen permanente, quedará un sistema de ecuaciones compatible determinado, por lo que se podrán establecer todos los parámetros empleados en el modelo.

Tabla 1: Valores en régimen permanente para pulso (HR), presión sistólica (PS) y presión diastólica (PD) para dos condiciones de inmersión.

\begin{tabular}{|c|c|c|}
\cline { 2 - 3 } \multicolumn{1}{c|}{} & Variable & $\begin{array}{c}\text { Valor en régimen } \\
\text { permanente }\end{array}$ \\
\hline \multirow{3}{*}{ No inmersión } & $\mathrm{HR}[\mathrm{lpm}]$ & 74 \\
\cline { 2 - 3 } & $\mathrm{PS}[\mathrm{mmHg}]$ & 110 \\
\cline { 2 - 3 } & $\mathrm{PD}[\mathrm{mmHg}]$ & 76 \\
\hline \multirow{2}{*}{$\begin{array}{c}\text { Inmersión hasta } \\
\text { el cuello }\end{array}$} & $\mathrm{HR}[\mathrm{lpm}]$ & 70 \\
\cline { 2 - 3 } & $\mathrm{PS}[\mathrm{mmHg}]$ & 122 \\
\cline { 2 - 3 } & $\mathrm{PD}[\mathrm{mmHg}]$ & 80 \\
\hline
\end{tabular}

\section{RESULTADOS}

El modelo propuesto se ha implementado en MATLAB-Simulink, y se ha simulado el sistema para un varón de $1.70 \mathrm{~m}$ de altura y $70 \mathrm{~kg}$ de masa corporal. La Tabla 2 resume las salidas del sistema para diferentes niveles de inmersión. Además, la Figura 3 muestra la entrada del sistema y la evolución según el nivel de inmersión de la elasticidad venosa, la distribución sanguínea, las acciones de regulación del ritmo cardíaco, el pulso y la MAP junto con la presión arterial media percibida por los barorreceptores.

Cabe destacar que los niveles de inmersión se han ajustado para representar inmersiones hasta el cuello $(144.50 \mathrm{~cm})$ y hasta la apófisis xifoides $(118.50 \mathrm{~cm})$, de acuerdo con la altura del usuario y medidas estándar del cuerpo humano.

\section{DISCUSIÓN}

Con este modelo lo que se pretende es estimar el estado del usuario, buscando estudiar y comprender los cambios en el sistema cardiovascular producidos por la inmersión en agua termoneutral $\left(34,5^{\mathrm{O}} \mathrm{C}\right)$. Por ello, cabe destacar que, aunque algunas variables presenten sobreoscilaciones por la naturaleza del sistema, para nuestro caso estamos especialmente interesados en estudiar el régimen permanente.

Uno de los principales efectos producidos cuando el cuerpo humano se sumerge es el decremento de la elasticidad de las venas, causada por la compresión hidrostática (Figura 3.b). Esto causa que una cierta cantidad de sangre se redistribuya desde los miembros inferiores hasta la cavidad torácica, especialmente al circuito pulmonar (Figura 3.c) .

Esta redistribución provoca ciertos cambios en el volumen sistólico, lo que conlleva un aumento del gasto cardíaco, estimado multiplicando el $H R$ por $Q_{L}$ (Tabla 2). Además, esto vendrá acompañado

Tabla 2: Valores en régimen permanente para diferentes niveles de inmersión, calculados para un varón de $170 \mathrm{~cm}$ de altura y $70 \mathrm{~kg}$.

\begin{tabular}{llll}
\hline Variable & Condición 0 & Condición 1 & Condición 2 \\
\hline Nivel de inmersión $(\mathrm{cm})$ & 0.00 & 144.50 & 118.50 \\
\hline Pulso (lpm) & 74.00 & 70.00 & 71.74 \\
\hline Gasto cardíaco $(\mathrm{mL} / \mathrm{min})$ & 5032.00 & 5880.00 & 5436.50 \\
\hline Presión sistólica $(\mathrm{mmHg})$ & 110.00 & 122.00 & 116.85 \\
\hline Presión diastólica $(\mathrm{mmHg})$ & 76.00 & 80.00 & 78.56 \\
\hline MAP (mmHg) & 87.30 & 93.72 & 90.29 \\
\hline MAP percibida por barorreceptores $(\mathrm{mmHg})$ & 87.30 & 94.00 & 91.33 \\
\hline Presión auricular derecha $(\mathrm{mmHg})$ & 5.13 & 9.12 & 8.08 \\
\hline Presión auricular izquierda $(\mathrm{mmHg})$ & 6.45 & 10.61 & 9.43 \\
\hline Volumen arterias $(\mathrm{mL})$ & 504.16 & 512.16 & 509.29 \\
\hline Volumen venas $(\mathrm{mL})$ & 2486.70 & 1978.70 & 2214.82 \\
\hline Elasticidad venas $(\mathrm{mL} / \mathrm{mmHg})$ & 150.00 & 29.58 & 51.26 \\
\hline Resistencia periférica $(\mathrm{mmHg} \mathrm{cdot} \mathrm{ms} / \mathrm{mL})$ & 1041.34 & 959.18 & 997.54 \\
\hline Volumen sistólico izquierdo $(\mathrm{mL})$ & 68.00 & 84.00 & 76.57 \\
\hline Volumen sistólico derecho $(\mathrm{mL})$ & 68.00 & 84.00 & 76.57 \\
\hline Circulación periférica $(\mathrm{mL})$ & 68.00 & 84.00 & 76.57 \\
\hline
\end{tabular}



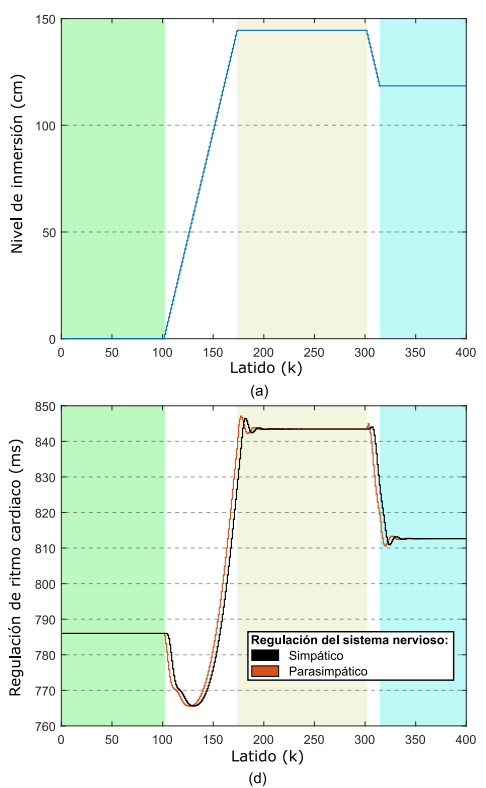
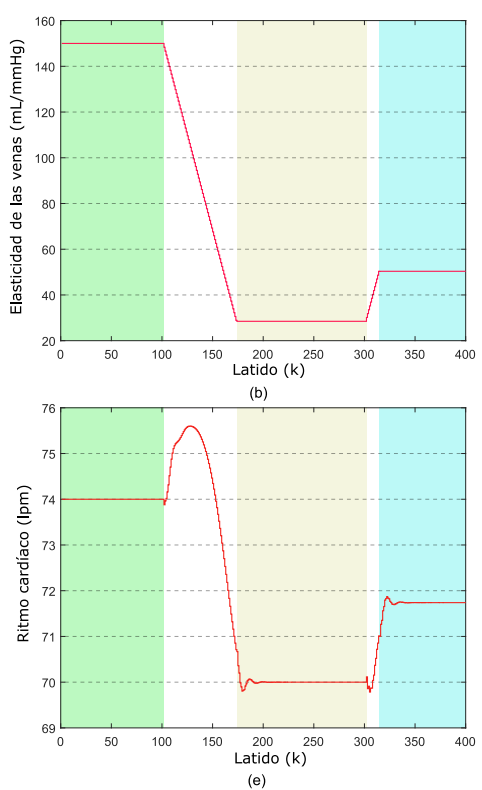
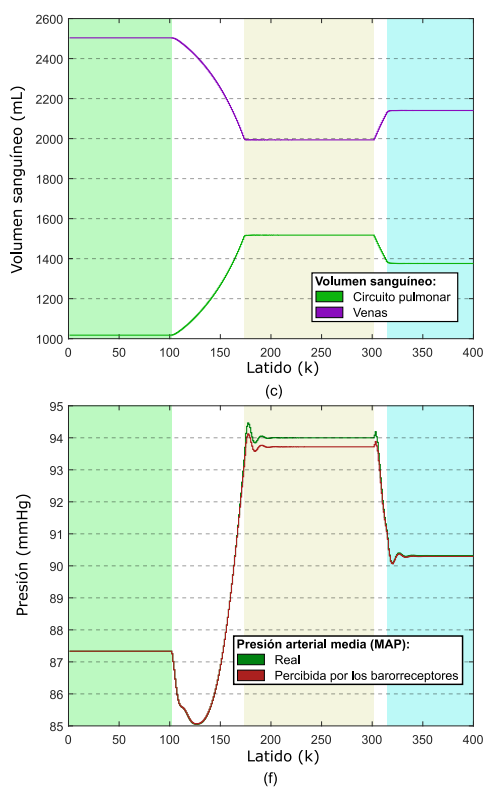

Figura 3: Resultados obtenidos en simulación para un varón de $170 \mathrm{~cm}$ de altura y $70 \mathrm{~kg}$. Cada sección coloreada representa un nivel de inmersión: en verde no inmersión, en naranja inmersión hasta el cuello, en azul inmersión hasta la apófisis xifoides (a) Nivel de inmersión, introducido como entrada del sistema (b) Evolución de elasticidad de las venas según el nivel de inmersión (c) Evolución de la distribución sanguínea en venas y circuito pulmonar (d) Regulación del pulso por el sistema nervioso simpático y parasimpático, empleadas como acción de control del sistema (e) Evolución del pulso según el nivel de inmersión (f) Presión arterial media y presión arterial media percibida por los barorreceptores

por un decremento en la resistencia periférica.

Si observamos las presiones en la Tabla 2 se observa un incremento de las presiones sanguíneas durante la inmersión, cuyo efecto se reportó en el estudio elaborado por K. S. Park et al. Además, cabe destacar el efecto del modelado sigmoidal de la sensibilidad de los barorreceptores (Figura 3.d). Se observa que, a mayor MAP, la sensibilidad de los barorreceptores decae, por lo que perciben una menor presión sanguínea.

Por otra parte, existe un decremento del ritmo cardíaco durante la inmersión (Figura 3.e). A pesar de que K. S. Park et al. no obtienen resultados significativos, otros estudios recogidos en el trabajo de I. M. Wilcock et al. observan una cierta tendencia del pulso a disminuir.

Como se ha mencionado previamente, el reflejo barorreceptor será el encargado de variar tanto el pulso como la resistencia periférica para así regular la presión sanguínea. Tanto el sistema nervioso simpático como el parasimpático tendrán influencia sobre el pulso. Estas acciones de regulación se muestran en la Figura 3.d, donde se observa un cierto retardo de la señal simpática con respecto a la parasimpática, debido a que las acciones del sistema nervioso simpático son más lentas. Además, cabe mencionar que, para mayor presión sanguínea, se observa una acción de control que tiende a aumentar el intervalo R-R, disminuyendo de esta forma el ritmo cardíaco.

\section{CONCLUSIÓN}

Se ha presentado un modelo matemático para tratar de comprender y predecir las respuestas del sistema cardiovascular durante la inmersión en un entorno acuático. Se demuestra mediante simulación que el modelo es capaz de predecir correctamente un considerable número de efectos producidos para diferentes niveles de inmersión, de acuerdo con los resultados aportados por otros autores.

A pesar de que el modelo ha sido evaluado en simulación, se pretende validar el modelo propuesto en una sesión experimental, examinando si es capaz de predecir correctamente las respuestas del sistema cardiovascular para diferentes niveles de inmersión. Asimismo, como planteamos emplear el modelo como una herramienta para predecir el estado del usuario durante escenarios de rehabilitación, se investigará la posibilidad de incorporar al modelo las respuestas producidas por un cambio de temperatura del agua, así como los efectos producidos al realizar un ejercicio de rehabilitación, tanto de forma asistida como no asistida. 


\section{Agradecimientos}

Este trabajo ha sido subvencionado por el Ministerio de Ciencia e Innovación, perteneciente a la Agencia Estatal de Investigación (AEI), a través del proyecto PID2019-108310RB-I00/AEI/10.13039/

501100011033 .

\section{English summary}

\section{CARDIOVASCULAR PREDICTIVE MODEL DURING WATER IMMERSION}

\begin{abstract}
The cardiovascular system is affected when the human body is immersed in water. To understand and predict the effects on the cardiovascular system during immersion, a high-order model is presented. The model describes the cardiovascular responses according to immersion level with a set of difference equations. The model is evaluated in simulation, and the simulated responses are compared to effects reported in the scientific literature in experimental scenarios
\end{abstract}

Keywords: Cardiovascular modelling, rehabilitation robotics, aquatic rehabilitation.

\section{Referencias}

[1] DeBoer, R. W., Karemaker, J. M., \& Strackee, J. (1987). Hemodynamic fluctuations and baroreflex sensitivity in humans: a beat-to-beat model. American Journal of Physiology-Heart and Circulatory Physiology, 253(3), H680-H689.

[2] Gisler, S. (2011). Modelling and control of the human cardiovascular system (Master's thesis, Eidgenössische Technische Hochschule Zürich, Sensory-Motor Systems Lab (SMS)).

[3] Greenway, C. V., \& Lautt, W. W. (1986). Blood volume, the venous system, preload, and cardiac output. Canadian journal of physiology and pharmacology, 64(4), 383-387.
[4] Guyton, A. C., \& Hall, J. E. (2006). Medical physiology. Gökhan N, Çavuşoğlu H (Çeviren), 3.

[5] Hill, L. K., Sollers III, J. J., \& Thayer, J. F. (2013). Resistance reconstructed: Estimation of total peripheral resistance from computationally-derived cardiac output. Biomedical sciences instrumentation, $49,216$.

[6] Kesiktas, N., Paker, N., Erdogan, N., Gülsen, G., Biçki, D., \& Yilmaz, H. (2004). The use of hydrotherapy for the management of spasticity. Neurorehabilitation and neural repair, 18(4), 268-273.

[7] McDowall, L. M., \& Dampney, R. A. (2006). Calculation of threshold and saturation points of sigmoidal baroreflex function curves. American Journal of Physiology-Heart and Circulatory Physiology, 291(4), H2003-H2007.

[8] Miller, E. L., Murray, L., Richards, L., Zorowitz, R. D., Bakas, T., Clark, P., \& Billinger, S. A. (2010). Comprehensive overview of nursing and interdisciplinary rehabilitation care of the stroke patient: a scientific statement from the American Heart Association. Stroke, 41(10), 2402-2448.

[9] Nadler, S. B., Hidalgo, J. U., \& Bloch, T. (1962). Prediction of blood volume in normal human adults. Surgery, 51(2), 224-232.

[10] Nas, K., Yazmalar, L., Şah, V., Aydın, A., \& Öneş, K. (2015). Rehabilitation of spinal cord injuries. World journal of orthopedics, $6(1), 8$.

[11] Park, K. S., Choi, J. K., \& Park, Y. S. (1999). Cardiovascular regulation during water immersion. Applied Human Science, 18(6), 233-241.

[12] Rekand, T., Hagen, E. M., \& Grønning, M. (2012). Spasticity following spinal cord injury. Tidsskrift for Den norske legeforening.

[13] Risch, W. D., Koubenec, H. J., Beckmann, U., Lange, S., \& Gauer, O. H. (1978). The effect of graded immersion on heart volume, central venous pressure, pulmonary blood distribution, and heart rate in man. Pflügers Archiv, 374(2), 115-118.

[14] Roper, J. A., Bressel, E., \& Tillman, M. D. (2013). Acute aquatic treadmill exercise improves gait and pain in people with knee osteoarthritis. Archives of physical medicine and rehabilitation, 94(3), 419-425. 
[15] Thibaut, A., Chatelle, C., Ziegler, E., Bruno, M. A., Laureys, S., \& Gosseries, O. (2013). Spasticity after stroke: physiology, assessment and treatment. Brain injury, 27(10), 1093-1105.

[16] Timischl, S. (1998). A global model for the cardiovascular and respiratory system. Karl-Franzens-Universität Graz, Austria.

[17] Veerbeek, J. M., Langbroek-Amersfoort, A. C., Van Wegen, E. E., Meskers, C. G., \& Kwakkel, G. (2017). Effects of robot-assisted therapy for the upper limb after stroke: a systematic review and meta-analysis.
Neurorehabilitation and neural repair, 31(2), 107-121.

[18] Wilcock, I. M., Cronin, J. B., \& Hing, W. A. (2006). Physiological response to water immersion. Sports medicine, 36(9), 747-765.

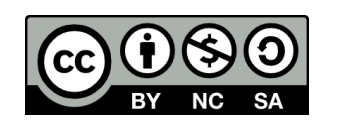

(C) 2021 by the authors. Submitted for possible open access publication under the terms and conditions of the Creative Commons Attribution CC BY-NC-SA 4.0 license (https://creativecommons.org/licenses/by-nc-sa/4.0/deed.es). 